\title{
A Vendor-Independent Resource Control Framework for WiMAX
}

\author{
Pedro Neves ${ }^{1}$, Tuomas Nissilä ${ }^{2}$, Telmo Pereira ${ }^{3}$, Ilkka Harjula ${ }^{2}$, João Monteiro ${ }^{3}$, \\ Kostas Pentikousis ${ }^{2}$, Susana Sargento ${ }^{3}$, and Francisco Fontes ${ }^{1}$ \\ ${ }^{1}$ Portugal Telecom Inovação, Rua Eng. José Ferreira P. Basto 3810-106 Aveiro, Portugal \\ \{est-p-neves,fontes\}@ptinovacao.pt \\ ${ }^{2}$ VTT Technical Research Centre of Finland, Kaitoväylä 1, FI-90571 Oulu, Finland \\ \{tuomas.nissila, ilkka.harjula, kostas.pentikousis\}@vtt.fi \\ ${ }^{3}$ Instituto de Telecomunicações, Campus Universitário Santiago, 3810-193 Aveiro, Portugal \\ \{tpereira,jmonteiro\}@av.it.pt, ssargento@det.ua.pt
}

\begin{abstract}
In this paper, a novel solution to dynamically control the resources of a WiMAX system is proposed. The presented solution is aligned with the NGN trends, as well as with the WiMAX Forum guidelines and architecture. The interaction with the WiMAX systems is performed through SNMP, supporting the standardized IEEE 802.16f MIB. Furthermore, the paper defines an horizontal abstraction layer, hiding the WiMAX equipments specific functionalities from the network control plane, thus providing robustness and vendor independency. In order to verify the performance of the proposed framework, a WiMAX testbed has been installed, and a set of measurements have been made, showing that the proposed system ensures a quick resource allocation, even for a large number of simultaneous resource reservation requests.
\end{abstract}

\section{Introduction}

The Access Network (AN), which is the important path between the operator Core Network (CN) and the Customer Premises Network (CPN), often has high requirements with respect to available bandwidth, and must be thoroughly planned. Current wired solutions, such as coaxial cable, Digital Subscriber Line (DSL) and fiber, are the main access technologies used for "last mile" connectivity. Nevertheless, due to the associated costs and difficult terrestrial conditions, important geographical areas are left without broadband connectivity. Often, lack of broadband access is the main reason for unequal access to Information and Communication Technologies (ICTs), a gap known as the Digital Divide. Previously, a significant amount of effort and resources have been invested in broadband wireless access technologies (e.g. Motorola Canopy) in order to fill this gap. Yet, since these were proprietary solutions, their adoption has been very low.

IEEE 802.16, a Broadband Wireless Access (BWA) solution for Wireless Metropolitan Area Networks (WMANs), covering large distances with very high throughputs, is an attractive BWA technology that can be used to overcome the previously mentioned limited access to the ICTs, for both fixed and mobile environments [1] [2]. However, the IEEE 802.16 standards do not define an end-to-end (E2E) system or architecture. They only define the PHY and MAC layers in the protocol stack. The Worldwide Interoperability for Microwave Access (WiMAX) Forum [3] [4] is currently defining a high-performance “All-IP" E2E network architecture to support fixed and mobile users. The WiMAX Forum is also committed to perform an interoperability effort across different vendors. This opens a different set of business opportunities for telecom operators, turning WiMAX into a viable technology for the Next Generation Network (NGN) environments [5] [6]. In these environments, users wish for having ubiquitous Internet access with a wide range of possible services (e.g. "triple play") and assured QoS guarantees, even while moving. These characteristics will lead NGNs to the ABC (Always Best Connected) paradigm, and as a consequence, operators have to cope with a whole new set of challenging requirements. Therefore, all the network design, including the WiMAX access network, should be able to address these aspects.

This paper presents a NGN architecture able to integrate an E2E QoS system in WiMAX environments, including support for real-time services, focusing on the resource management mechanisms. The framework for this work is the EU IST WEIRD project [7]. The network architecture in WEIRD takes into consideration standardization developments at 
fora such as IEEE, IETF 16ng [8] and the WiMAX Forum, as well as NGN trends. The results obtained from the development and experimental setup of this architecture show that the processing times for the resource reservations, both in the resource control modules and WiMAX equipments are small, even for a large number of simultaneous reservation requests, which enable the use of WiMAX in real-time environments with high mobility scenarios.

The paper is organized as follows. Section 2 addresses related work and section 3 describes our system architecture, focusing on the resource control modules. Section 4 details these modules that control the resources of the channel and integrate the WiMAX technology in the WEIRD environment, whereas Section 5 discusses the experimental results obtained, as well as our test methodology. Finally, Section 6 concludes this paper.

\section{Related Work}

There are some projects that work and propose new models for the support of QoS mechanisms in 802.16 technologies, such as IST FP6 AROMA [9], IST FP6 WISECOM [10], IST FP6 PLUTO [11] and SUIT [12]. However, none of them offer a truly paradigm to differentiate traffic, integrating WiMAX technology with IP networks and offering an E2E QoS support.

The EU IST DAIDALOS project [13] is also defining a NGN architecture, where the seamless integration of heterogeneous network technologies such as WiMAX, WiFi and UMTS, is envisaged. The defined architecture provides seamless QoS support using NSIS for QoS signaling, separating E2E QoS control at the network layer from link-local QoS at the layer below. Nevertheless, WEIRD is one step ahead, by defining a WiMAX vendor independent framework, that effectively controls all WiMAX network related functionalities, including QoS integration, admission control mechanisms and fast mobility issues. Furthermore, as in DAIDALOS, WEIRD also uses NSIS for network layer QoS signaling. Nonetheless, WEIRD has extended the generic NSIS QSPEC [14] objects to include specific WiMAX related QoS parameters, such as: Minimum Reserved Traffic Rate, Maximum Sustained Traffic Rate, Scheduling Class, Unsolicited Grant Interval, Minimum Latency and Tolerated Jitter.

\section{WEIRD Architecture}

The WiMAX Forum defines an E2E IP framework that aims at ensuring full interoperability between Base Stations (BSs) and Mobile/Subscriber Stations (MSs/SSs) from different vendors. The WiMAX
Forum extends IEEE $802.16 \mathrm{~d} / \mathrm{e}$ by defining a Network Reference Model (NRM), a logical representation of the WiMAX network architecture, based on a set of functional entities (FEs) and eight standardized interfaces, known as Reference Points (RPs), named "R1", "R2”, ..., "R8”. Using this model, multiple implementation options for each FE are allowed, while maintaining interoperability using the RPs.

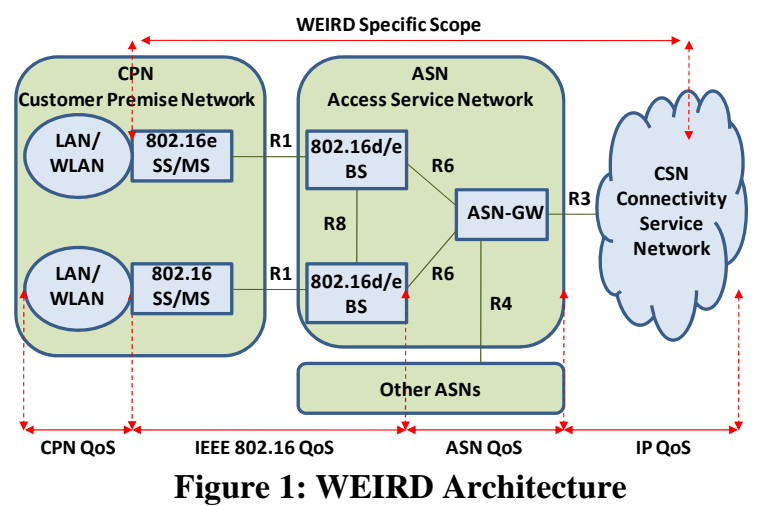

Figure 1 illustrates the WEIRD overall architecture, based on the WiMAX Forum NRM. Three functional entities are defined: Connectivity Service Network (CSN), Access Service Network (ASN) and the Customer Premise Network (CPN). The CPN is the terminal equipment, responsible for establishing radio connectivity with the BS. For IEEE 802.16d, the CPE might be composed by single-user SSs or multi-users SSs (MSS) when a LAN/WLAN is connected to the SS. Regarding IEEE 802.16e, a single-user Mobile Station (MS) is the envisaged scenario. The ASN is generally composed by several BSs connected to several gateways (ASN-GWs), which establish connectivity with the CSN. The ASN includes a set of functionalities in order to provide radio connectivity to WiMAX subscribers. Additionally, it also performs relay functions to the CSN in order to establish IP connectivity and Authentication, Authorization and Accounting (AAA) mechanisms. Finally, the CSN holds the DHCP, DNS and AAA servers, as well the Application Function (AF) - in this case the SIP Proxy. Moreover, the CSN is responsible for establishing connectivity with the IP backbone.

Following NGN trends [15], the WEIRD system [16] follows a multi-plane structure. Vertically it is composed by two stratums - the Applications and Service Stratum responsible for the management and control of the applications and the Transport Stratum responsible for the resources management, as well as the data transport. Horizontally, the WEIRD 
architecture is split into three well-known parallel planes - Management, Control and Data Plane.

Figure 2 depicts the WEIRD Control Plane modules and interfaces. Although NRM is used as the basis of the architecture, new modules have been defined on the Control Plane to efficiently support real time services with QoS differentiation. Both SIP [17] and legacy applications are supported. For SIP-based applications, the SIP User Agent (SIP UA) in the MS communicates directly with the SIP Proxy at the CSN. For legacy applications, a specific module is specified for the MS - the WEIRD Agent - which adapts and configures the QoS parameters as required by legacy applications.

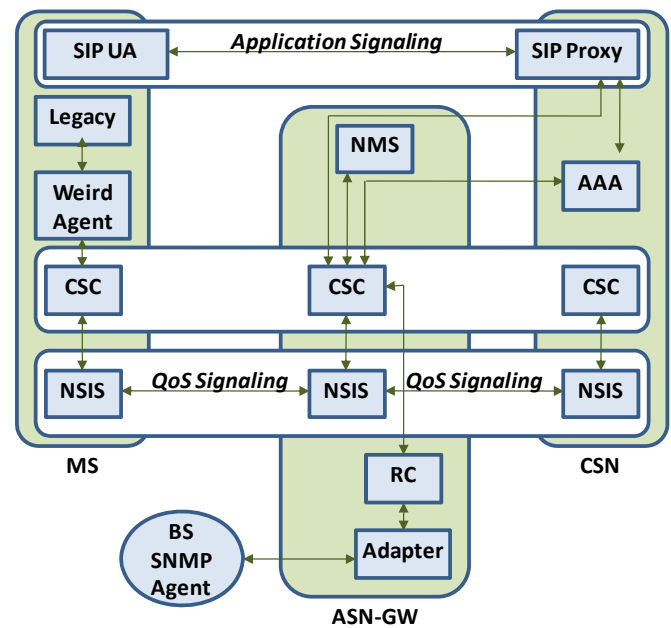

Figure 2: WEIRD Architecture - Control Plane

The Connectivity Service Controller (CSC) modules, located at all NRM entities, include the most important functions of the system. Since WEIRD is focused on the ASN segment, the CSC at the ASN (CSC_ASN) is the main coordination point for QoS functions, such as resource allocation and admission control in the ASN and the WiMAX segments. For SIP applications, the SIP Proxy extracts the QoS parameters from the SIP/SDP messages, performs user authentication and authorization with the AAA server, and forwards the collected QoS information to the CSC_ASN using a Diameter (Gq/Gq') [18] interface.

CSC_MS communicates with the WEIRD Agent to obtain the QoS parameters required by the legacy applications and provides this information to the main QoS coordination point (CSC_ASN). When the CSC_CSN receives the QoS reservations requests from the CSC_ASN, it establishes the QoS paths on the core network. Moreover, the CSC_ASN has an interface with the Network Management System (NMS) for medium- and long-term functions, such as QoS provisioning.

The communication between the several CSCs (MS, ASN and CSN) is performed through the usage of the Next Steps in Signaling (NSIS) QoS signaling protocol [19]. NSIS decomposes the overall signaling protocol suite into a generic (lower) layer and specific upper layers for each specific signaling application. At the lower layer, General Internet Signaling Transport (GIST) [20] offers transport services to higher layer signaling applications. Above this layer, the NSIS Signaling Layer Protocol (NSLP) [21] supports any protocol within the signaling application layer.

All functions related with the WiMAX system are managed and controlled by the Resource Controller (RC), which can be seen as the WiMAX link manager. The $R C$ is responsible for the QoS management in the WiMAX link, including Service Flows (SFs) and Convergence Sublayer classifiers control (including Ethernet, IPv4 and IPv6 classification), as well as for admission control tasks on the WiMAX link. Furthermore, the $R C$ acts as an abstraction layer between the upper parts of the architecture and the lower level modules. It hides all WiMAX technology related functionalities from the upper layers, keeping them independent and oblivious of WiMAX-specific QoS characteristics. To enforce the QoS decisions on the WiMAX BS, the $R C$ triggers the Adapter module that will communicate the decisions to the WiMAX BS through an SNMP interface. The initial proposed model of WiMAX Adapter is presented in [22]. It is split into a Generic Adapter (GA) component and one or more Vendor-Specific Adapter (VSA) modules.

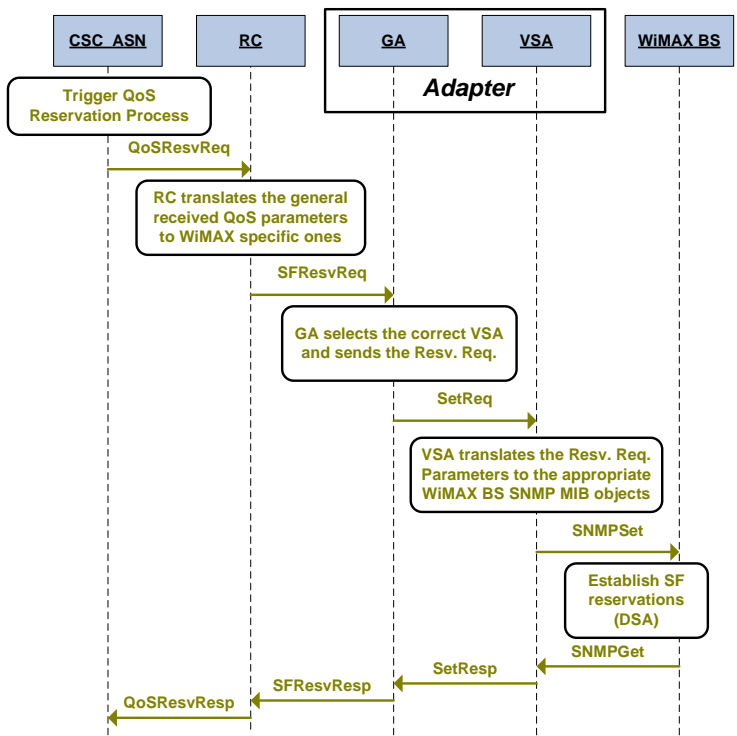

Figure 3: QoS reservation signaling diagram 
With this approach, different WiMAX equipments can be integrated into the WEIRD architecture by developing a VSA for each new vendor equipment, if necessary. From all modules defined on the WEIRD architecture, VSAs are the only dependent on the specific WiMAX equipment used. As an example, Figure 3 illustrates the signaling between the different entities involved in the QoS reservation process. The signaling for QoS modifications and deletions is similar.

\section{Resource Control}

Figure 4 depicts the resource control modules, described in the following subsections, which implement our proposed framework.

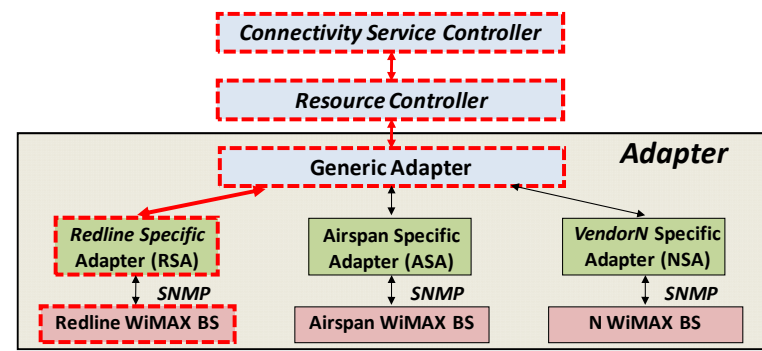

Figure 4: Developed Framework

\subsection{Resource Controller}

The $R C$, located at the ASN-GW, has been defined and implemented in order to provide an abstraction layer that seamlessly supports WiMAX technology within the WEIRD NGN architecture. Moreover, the $R C$ also works as the QoS manager for the WiMAX network, handling the creation, modification and deletion of SFs, as well as providing full control of all the Service Classes (SCs) and the related Convergence Sublayer classifiers. Finally, another important feature of the RC is the capability to distinguish between Mobile and Fixed WiMAX equipments, and to proceed accordingly. As defined in the IEEE $802.16 \mathrm{~d} / \mathrm{e}$ standard, three different classes of SFs are distinguished and supported by the $R C$ : (a) Provisioned Service Flows which are created via NMS provisioning; (b) Admitted Service Flows which have their resources reserved by the BS, but are not yet active; and (c) Active Service Flows, which have their resources committed and ready to be used for data transfer.

With respect to the Convergence Sublayer classifiers, the $R C$ currently supports Ethernet, IPv4 and IPv6 CSs. To manage SFs and their status, both Static and Dynamic models have been defined and implemented at the $R C$. The Static model is used for medium- and long-term QoS reservations, triggered by the NMS. In this case, the SF must traverse all the status (Provisioning and Admitted) until it reaches the Active one. Under the Dynamic model, the SF can be directly activated without traversing the remaining status. This model is suitable for real-time services that require instantaneous allocations of SFs on the WiMAX path.

\subsection{The WEIRD Adapters}

An initial model for the WEIRD Adapter was suggested in [22]. In this work we concentrate on the proposed ASN-GW Adapter, using the SNMP [23] network management interface of the WiMAX equipment. To cope with the complexity that the increasing heterogeneity brings into future communication networks, we propose a common solution that applies to different vendor equipment, and further on a common solution that fits different technologies.

\subsubsection{Generic Adapter}

The $G A$ allows the convergence between different VSAs for the adaptive applications and processes, such as the Resource Controller. GA provides an abstraction of the hardware management functions. All details are hidden from the upper modules, allowing the use of common primitives. To allow this, the GA converts the common primitives to more specific primitives, serving the needs of the Specific Adapters. The GA has been implemented as a Linux user-space module, using ANSI $\mathrm{C}$ and standard libraries. $G A$ is able to communicate with local and remote VSAs modules using inter-process communication. The $R C$ and any other adaptive application/process [22] can also be local or remote user of the $G A$ module.

\subsubsection{Vendor Specific Adapters}

The VSA deals with the WiMAX equipment specificities. For each vendor-specific equipment, a library is implemented providing a differentiated request processing. In our case, the Airspan Specific Adapter (ASA) has been implemented to interface with Airspan WiMAX equipment, and the Redline Specific Adapter (RSA) has been implemented to interface with the Redline Communications WiMAX equipment.

Each VSA is responsible to fetch information from the WiMAX network elements (allowing important control functions, such as admission control and effective resources control) and to enforce the control decisions triggered by the CSC and $R C$ in the WiMAX segment. Hence, its functionalities assume considerable relevance in the global architecture.

ASA is designed to deal with the SNMP management actions of the Airspan WiMAX BS 
equipment. In the Airspan BS equipment, the implemented SNMP features allow to retrieve SNMP monitoring data for different monitoring purposes, including system description, system capability, network statistics and topology information. ASA is implemented using the Net-SNMP C API.

The RSA interfaces with the Redline Communications WiMAX equipment enforcing QoS requests from the upper layer modules in the 802.16 network, respecting completely the 802.16f [24] standard, and retrieving resource and topology information. This information is crucial for the effective implementation of resource control mechanisms. RSA uses the SNMP protocol when communicating with the SNMP Agent module, and alternatively the CLI/TELNET interface to obtain the required information when the SNMP interface is not available. The SNMP interface was implemented using Net-SNMP API [25], and CLI/TELNET interface used a new particular solution with automated scripts. The $R S A$ is responsible for three main functionalities:

Service Flow Management (SFM) refers to managing and enforcing the received QoS reservations, modifications and deletions requests in the Redline WiMAX equipment, either using IPv4 or 802.3 CSs.

Resources Management (RM) shows in the aptitude to answer upon resource requests collecting information from the 802.16 network as RF, PHY and MAC parameters, including downlink and uplink available bandwidth for the SSs, and packing that information to the higher layer management entities.

Dynamic 802.16 Network Discovery (D16ND) consists on the ability to detect new SSs and BSs that join the WiMAX network. The complete network topology of the WiMAX segment is built in a dynamic way, supplying at the same time relevant PHY and MAC parameters to the NMS. This WiMAX network discovery feature is implemented over the SNMP traps supported by the Redline WiMAX equipment.

\section{Evaluation}

We tested the proposed resource control framework in the Portuguese (for the RSA) and Finnish (for the ASA) testbeds, based on the NRM, as illustrated in Figure 5. Particularly, the testbed is composed by the CSN, the ASN and the CPN. Under the ASN we have the BS directly connected to the ASN-GW. Two SSs are connected to the BS creating a Point-to-Multipoint (PMP) topology for the Radio Access Network (RAN).

Our tests focus on evaluating our system with respect to efficiently managing the WiMAX system and integrating it with a NGN IP architecture.
Specifically, we evaluate the performance of our architecture regarding QoS integration in a WiMAX RAN. Each module in the chain has been evaluated, as well as the overall path towards the WiMAX system. In Figure 4, using dashed red boxes, it is illustrated the system modules that have been tested (CSC, RC, GA and the RSA).

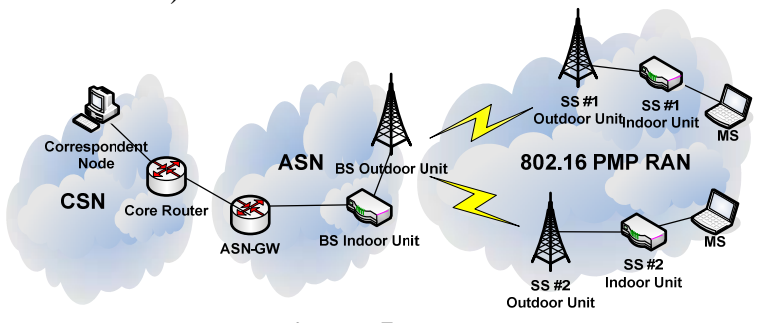

Figure 5: Testbed

The Redline Communications certified WiMAX equipment has been used since it fully supports the QoS actions that are evaluated: reservations, modifications and/or deletions. The results obtained for every specific QoS action that has been evaluated for a specific number of SFs (2, 8, 32, 64, 128 and 256) in both uplink and downlink directions, comprise the mean of three runs.

\subsection{Overall QoS Tests}

This section presents the results of QoS sessions establishments, modifications and deletions. Figure 6 illustrates a stacked column graphic, where each column represents a specific module of the ASN-GW (CSC, RC, GA and RSA).

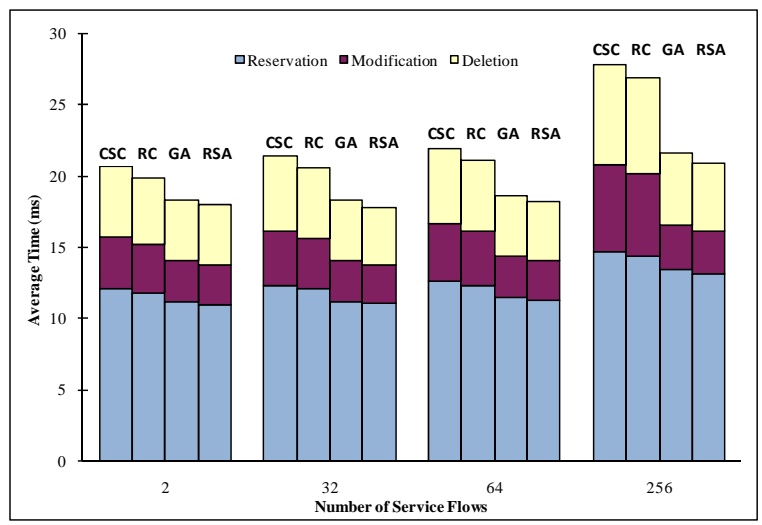

Figure 6: QoS reservation, modification and deletion measurements

Each stack column is further split in three parts, each one corresponding to a specific action, namely, QoS session establishment (blue), modification (purple) and deletion (white). For each action, the vertical axis represents the cumulative average time (in 
milliseconds - $m s$ ) to enforce a specific action on the WiMAX system. As an example, the blue part of the CSC column represents the cumulative average time, composed by the CSC, RC, GA, RSA and WiMAX BS modules, to establish the SF reservations in the WiMAX system. The blue part of the $R C$ column describes the QoS reservation cumulative average time, composed by the RC, GA, RSA and WiMAX BS, whereas the blue section of the GA column provides the cumulative average time, given by the GA, RSA and WiMAX BS modules. Finally, the RSA column represents the internal module processing time, as well as the SNMP management messages exchange with the WiMAX BS. On the horizontal axis is represented the number of SFs $(2,8,32,64,128,256)$ that have been used for each test. Therefore, each group of four columns represents a specific performance test.

Comparing the obtained results with respect to the type of actions that are being requested to the WiMAX system, we show that the needed time to establish a QoS reservation (blue) is approximately $53 \%$ of the total time consumed. The rationale for this behavior is due to the fact that the amount of operations required by the WiMAX system for a QoS reservation is higher compared with the modification and deletion processes. The figure also shows that the most time consuming module is the $R S A$, mainly because it includes the negotiation of the QoS parameters between the BS and the SS through the usage of the DSA/C/D (Dynamic Service Addition / Change / Deletion) messages, as defined in the IEEE 802.16 standard, which is the major time consuming process in the chain. The average time taken by the RSA to perform the three actions has a minimum of $17,9 \mathrm{~ms}$ for 2 SFs up to a maximum of $20,9 \mathrm{~ms}$ for $256 \mathrm{SFs}$, with small impact on the increase of SFs. The time spent by the remaining modules is due to the message flow and internal processing. The average time measured on the CSC to reserve $256 \mathrm{SFs}$ is $15 \mathrm{~ms}, 6$ $m s$ to modify the previously established SFs and then finally $6 \mathrm{~ms}$ to delete the $256 \mathrm{SF}$ reservations at the end of the flow lifetime. In this case, the entire management of the 256 flows required approximately $27 \mathrm{~ms}$.

In short, an increasing number of SFs slightly raises the time spent to establish, modify and delete the QoS sessions. This behavior occurs because the number of entries in the hash tables increases, as well as the interaction with the SNMP MIB tables in the WiMAX BS. The overall elapsed time is very good for real-time applications and fast mobility environments, ensuring a desirable quick resource reallocation, from $21 \mathrm{~ms}$ for 2 SFs up to $27 \mathrm{~ms}$ for $256 \mathrm{SFs}$.

\subsection{Redline Specific Adapter Tests}

This section is devoted to the discussion and analysis of the RSA performance results. A considerable array of times was collected in order to analyze the time distribution along the different tasks accomplished by $R S A$. The $R S A$, as described on subsection 4.2.2, is the module that interacts with the WiMAX equipment. Thus, the total time spent from the reception of a request (SF Reservation / SF Modification / SF Deletion) to the sending of the correspondent answer to the GA was obtained; the partial times were also gathered, in order to distinguish between the internal processing time of the RSA module and the time it takes to set each SNMP MIB [26] [27] table. Figure 7 illustrates the RSA performance times. The RSA Resv/Mod/Del Total Path blocks show the total time used up by RSA to process each request, that is, the entire path since it receives a request from the $G A$, until it sends back the response to the latter. The RSA Resv/Mod/Del Total SNMPSET blocks represent the sum of all SNMPSET times needed, respectively, for a SF reservation, modification, or deletion. Finally, the RSA Resv/Mod/Del Internal Processing blocks represent the internal processing time of RA routines.

As can be observed in Figure 7, the RSA internal processing time is so small that the total path time is almost not affected, and therefore unseen on the graphic bars. In the worst case, the internal processing time is less than 55 us. This case occurs when 256 SFs are deleted, which causes the module to deal with considerable processing work when looking for the associated indexes to set the SNMP MIB tables [24].

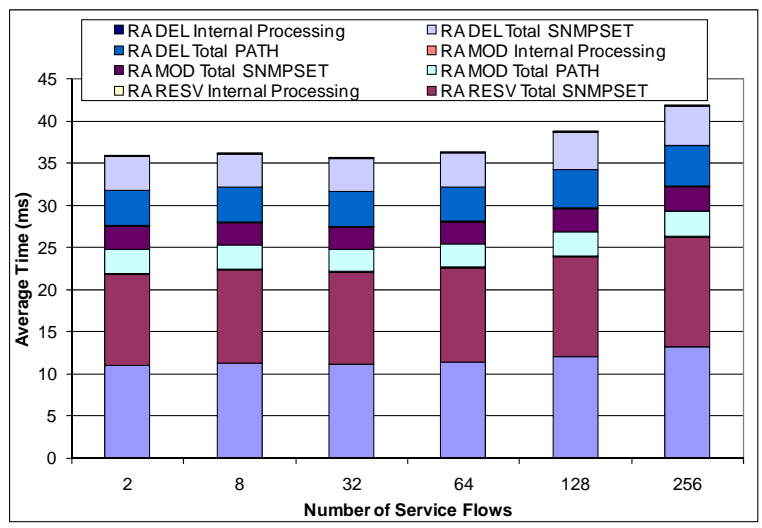

Figure 7: RSA performance times

Another important fact that can be concluded from the graphic is that QoS session's establishment takes more time than the deletion ones, and these, in turn, are more time consuming than the modification requests. Furthermore, we can also perceive that there is a slight 
increase of time when the number of SFs increases. Nevertheless, these values are kept stable, showing that the RSA and the WiMAX equipment are prepared to deal efficiently with a large number of sequential requests. Finally, the differences between the RSA Resv/Mod/Del Total SNMPSET processing times are due to the different amount of Object IDs (OIDs) that have to be set on the WiMAX MIB, which is larger for the reservation requests when compared to modifications or deletion ones.

Table I details all the SNMP MIB Tables, and the correspondent OIDs number that are assigned when performing a SF Reservation, Modification and Deletion.

Table I: WiMAX QoS MIB Tables

\begin{tabular}{|c|c|c|}
\hline SF Request: MIB Tables & Functionality & OIDs \\
\hline DEL : ServiceClassTable & \multirow{3}{*}{$\begin{array}{l}\text { Contains the SF } \\
\text { QoS parameters }\end{array}$} & 1 \\
\hline MOD : ServiceClassTable & & 5 \\
\hline RESV : ServiceClassTable & & 12 \\
\hline DEL : ProvisionedSfTable & \multirow{2}{*}{$\begin{array}{c}\text { Contains the SF } \\
\text { profiles provisioned by } \\
\text { NMS }\end{array}$} & 1 \\
\hline RESV : ProvisionedSfTable & & 5 \\
\hline RESV : ProvisionedForSFTable & $\begin{array}{c}\text { Maps the MAC } \\
\text { addresses of SSs to the } \\
\text { provisioned SFs }\end{array}$ & 1 \\
\hline RESV :ClassifierRuleTable & $\begin{array}{c}\text { Contains packet } \\
\text { classifier rules } \\
\text { associated with SFs }\end{array}$ & 14 \\
\hline
\end{tabular}

Figure 8 shows the time spent by every single SNMP MIB table set.

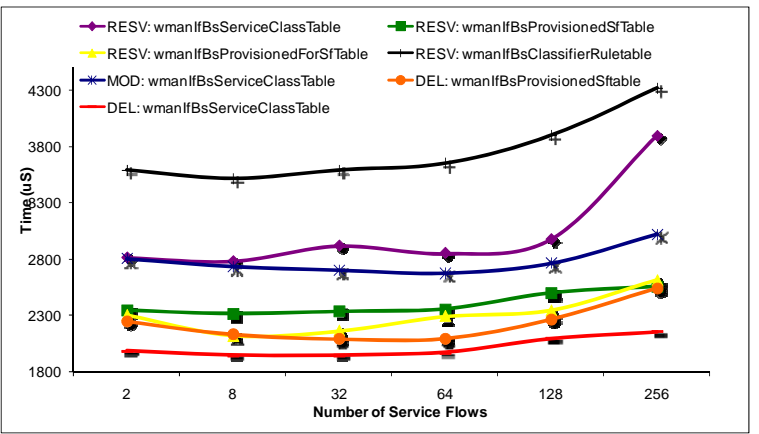

Figure 8: Single SNMPSET performance times

Once again, the inherent processing time is coupled with the increased number of SFs requests, staying a little higher as we increase the number of sequential requests. Furthermore, the time taken to process each SNMPSET is also strictly related with the number of OIDs that must be set in each operation - the greater the number of OIDs to assign, more time is spent on performing this task by the equipment. The differences that occur when setting distinct SNMP MIB tables with the same OIDs number are due to the inherent processing time of WiMAX equipment for those tasks.
For instance, erasing an existing SF by the WiMAX equipment is more time consuming than just doing some amendment to the QoS parameters of an existing one. Moreover, when deleting a ProvisionedSfTable row entry, we are in fact deleting, at the same time, all the associated entries in ProvisionedForSfTable and ClassifierRuleTable. For that reason the deletion process when erasing elements of ServiceClassTable is smaller than when deleting elements from ProvisionedSfTable.

\section{Conclusion and Future Work}

In this paper we proposed a novel solution for the dynamic resource control in WiMAX environments, motivating the use of the IEEE 802.16f MIB standard. The presented system architecture was developed in the WEIRD project, and comprises Resource Controller and WiMAX Adapter modules for the support of QoS reservations, modifications and deletions. To cope with the differences between different WiMAX manufacturers', the WiMAX Adapter was divided into a Generic Adapter and a Vendor Specific Adapter. This feature allows different WiMAX equipments to be integrated into the WEIRD architecture by developing only a Vendor Specific Adapter.

In order to validate the QoS resource management support of both Resource Controller and Adapters, QoS measurements have been performed in a WiMAX certified testbed. The achieved results have shown that the processing times for the QoS reservations, modifications and deletions are very small, enabling the use of our WiMAX based architecture in NGN real-time environments without traffic disruptions.

In the near future, we will evolve our testbed by adding Mobile WiMAX equipments. Thereafter, we will evaluate the developed framework performance in a miscellaneous Mobile and Fixed WiMAX environment. Furthermore, there is also motivation to extend the Resource Control solution to cope with different wired/wireless technologies, and not only with WiMAX systems. Having a common solution for different technologies in a single unit would be beneficial for the development of the future network management.

\section{Acknowledgment}

This work has been carried out in the framework of the IST Sixth Framework Programme Integrated Project WEIRD, which is partially funded by the Commission of the European Union (IST-034622-IP). The views expressed in this paper are solely those of the authors and do not necessarily represent the views 
of their employers, the WEIRD project, or the Commission of the European Union. We thank the partners of the WEIRD consortium for invaluable discussions.

\section{References}

[1] IEEE 802.16 Working Group, IEEE Standard for Local and Metropolitan Area Networks. Part 16: Air Interface for Fixed Broadband Wireless Access Systems, IEEE Std. 802.16-2004, October 2004.

[2] IEEE 802.16 Working Group, IEEE Standard for Local and Metropolitan Area Networks. Part 16: Air Interface for Fixed Broadband Wireless Access Systems. Amendment 2: Physical and Medium Access Control Layer for Combined Fixed and Mobile Operation in Licensed Bands, IEEE Std. 802.16e, December 2005.

[3] WiMAX Forum, WiMAX End-to-End Network Systems Architecture Stage 2: Architecture Tenets, Reference Model and Reference Points, Release 1.1.0, June 2007.

[4] WiMAX Forum, WiMAX End-to-End Network Systems Architecture Stage 3: Detailed Protocols and Procedures, Release 1.1.0, June 2007.

[5] ITU-T, General Overview of NGN, Rec. Y.2001, December 2004.

[6] ITU-T, General Principles and General Reference Model for Next Generation Networks, Rec. Y2011, October 2004.

[7] WiMAX Extension to Isolated Research Data networks (WEIRD) project: www.ist-weird.eu

[8] S. Madanapalli, Ed., Analysis of IPv6 Link Models for IEEE 802.16 Based Networks, IETF RFC 4968, August 2007.

[9] Advanced Resource Management Solutions for Future All IP Heterogeneous Mobile Radio Environments (AROMA) project: www.aroma-ist.upc.edu/

[10] Wireless Infrastructure over Satellite for Emergency Communications (WISECOM) project: www.wisecomfp6.eu/

[11] Physical Layer DVB Transmission Optimization (PLUTO) project: dea.brunel.ac.uk/pluto/

[12] Scalable, Ultra-fast and Interoperable Interactive Television (SUIT) project: suit.av.it.pt
[13] Designing Advanced network Interfaces for the Delivery and Administration of Location independent, Optimised Personal Services (DAIDALOS) project: www.ist-daidalos.org

[14] A. Bader, C. Kappler, D. Oran, QoS NSLP QSPEC Template, IETF NSIS WG Internet-Draft, July 2007.

[15] K. Knightson, N. Morita, T. Towle, NGN Architecture: Generic Principles, Functional Architectures and Implementation, IEEE Communications Magazine, p. 49-55, October 2005.

[16] E. Guainella, E. Borcoci, M. Katz, P. Neves, M. Curado, F. Andreotti, E. Angori, WiMAX Extension to Isolated Research Data Networks, IEEE Mobile WiMAX world summit, March 2007, Orlando (Florida).

[17] J. Rosenberg, G. Camarillo, SIP: Session Initiation Protocol, IETF RFC 3261, June 2002.

[18] P. Calhoun, J. Loughney, Diameter Base Protocol, IETF RFC 3588, September 2003.

[19] R. Hancock et al, Next Steps in Signaling (NSIS): Framework, IETF RFC 4080, June 2005.

[20] H. Schulzrinne, R. Hancock, GIST: General Internet Signalling Transport, IETF NSIS WG Internet-Draft, July 2007.

[21] J. Manner, G. Karagiannis, NSLP for Quality-of-Service Signaling, IETF NSIS WG Internet-Draft, July 2007.

[22] T. Nissilä, J. Huusko, I. Harjula, and M. Katz, Adapter Implementation between WiMAX Specific Layers and Network/Application Layers, 1st BWA Workshop, Cardiff, Wales, September, 2007, pp. 328-333.

[23] J. Case, M. Fedor, A Simple Network Management Protocol (SNMP), IETF RFC 1157, May 1990.

[24] IEEE 802.16 Working Group, IEEE Standard for Local and Metropolitan Area Networks, Part 16: Air Interface for Fixed Broadband Wireless Access Systems - Amendment 1: Management Information Base, IEEE Std. 802.16f-2005, December 2005.

[25] Net-SNMP website - http://net-snmp.sourceforge.net

[26] J. Case, K. McCloghrie, M.Rose, Management Information Base (MIB) for the Simple Management Protocol (SNMP), IETF RFC 3418, December 2002

[27] K. McCloghrie, D. Perkins, J. Schoenwaelder, Structure of Management Information version 2, IETF RFC 2578, April 1999 